\title{
Seminare / Séminaires 2007
}

\section{Praxiseröffnung/-übernahme}

Themen (Details $\rightarrow$ www.fmhservices.ch) Juristische Aspekte - Ehe- und Erbrecht - Praxiseinrichtung - Praxisadministration - Unternehmensbewertung einer Arztpraxis - Finanzierung einer Arztpraxis - Versicherungen.

\section{Sponsoren}

Versicherung der Schweizer Ärzte, Schweizerische Ärzte-Krankenkasse, Druckerei Schmid-Mogelsberg, Labornetz daCapo, Unilabs SA, Labor medica, Kern Concept AG und EMH Schweizerischer Ärzteverlag AG.

\section{Daten}

$\begin{array}{lll}\text { K05 Donnerstag, 8. November } 2007 & \text { Hotel } \\ \text { Basel } & 9.00-16.30 \mathrm{Uhr} & \text { Victoria }\end{array}$

\section{Praxisübergabe}

\section{Hinweis}

Vor allem aus steuerplanerischer Sicht lohnt es sich, sich bereits frühzeitig (5-10 Jahre) mit diesem Thema auseinanderzusetzen.

\section{Themen (Details $\rightarrow$ www.fmhservices.ch)} Juristische Aspekte - Unternehmensbewertung einer Arztpraxis - Versicherungen - Steuern.

\section{Sponsoren}

Versicherung der Schweizer Ärzte, Schweizerische Ärzte-Krankenkasse, Labor medica und EMH Schweizerischer Ärzteverlag AG.

\section{Daten}

K10 Donnerstag, 15. November 2007 Hotel

\section{Buchführung und Steuern}

Themen (Details $\rightarrow$ www.fmhservices.ch) Buchführung (Massnahmen vor Praxiseröffnung/-übernahme, Standardkontenplan für Ärzte, Doppelte Buchführung, EDV-unterstützte Buchführungslösung), Steuern (Eintritt in die Selbständigerwerbstätigkeit, Steuerfallen und Steuerrisiken, optimierte Steuerplanung).

\section{Sponsor \\ EMH Schweizerischer Ärzteverlag AG.}

\section{Daten}
K14 Donnerstag, 22. November 2007 Hotel Basel 13.30-18.00 Uhr Victoria

\section{Praxiscomputerworkshop}

\section{Inhalt}

Unter der Leitung von SGAM.Informatics erhalten die Workshopteilnehmer/innen im 1. Teil eine Einführung in die Anforderungen eines Praxisinformationssystems. Anhand einer modernen vernetzten Praxisinfrastruktur werden die Beurteilungskriterien für eine praxis- und zukunftstaugliche Softwarelösung dargestellt. Checklisten sollen die schnelle Orientierung unterstützen und bei der Beurteilung und Wahl des Produkts konkrete Hilfe bieten. Der 2. Teil umfasst die Präsentation von sechs Praxisadministrationssoftwarelösungen (Leistungserfassung, elektronisches Abrechnen unter Einbezug der TrustCenter, Agendaführung, Statistiken, Laborgeräteeinbindung, elektronische Krankengeschichte, Finanzbuchhaltungslösungen usw.).

\section{Ziel}

Die Workshopteilnehmer/innen erhalten produkteunabhängige Beurteilungskriterien für ein modernes Praxisinformationssystem. Zudem erhalten sie einen ersten Überblick über führende Softwarelösungen.

\section{Daten}

K17 Donnerstag, 29. November 2007 Stadttheater Olten $\quad 9.30-16.30 \mathrm{Uhr} \quad$ Olten

\section{Folgende Softwareanbieter können Sie} im 2. Teil des Workshops kennenlernen:

\section{Ärztekasse, Urdorf (CB 6)}

Als Marktleader seit 1964 im Bereich Praxisadministration können Sie bei der Ärztekasse auf eine ganzheitliche Lösung für Ihre Fragen betreffend Abrechnungsvarianten, Computerwahl, Netzwerke, Formular- und Briefbearbeitung usw. zählen. Die statistischen Erhebungen (im Auftrag 
der FMH Roko) sind ein anerkanntes betriebswirtschaftliches und standesorganisatorisches Hilfsmittel für die moderne Praxisführung. Nebst innovativen Ideen und Lösungen sowie Kooperationspartnerschaft mit 10 TrustCentern ist uns eine umfassende Kundenbetreuung ein Anliegen. Unsere Standorte befinden sich in Basel, Bern, Chur, Crissier, Genf, Le Landeron, Lugano, Luzern, St. Gallen, Thônex und Zürich. Sie definieren Ihre Wünsche - wir bieten Ihnen die Lösung!

\section{Delemed AG, Kehrsatz (PEX II)}

Delemed AG entwickelt und vertreibt seit 15 Jahren erfolgreich PEX II, die einfache, sympathische und effiziente Software für die Arztpraxis und Kliniken. Durch einen modularen Aufbau kann das System jederzeit den aktuellen Bedürfnissen des Kunden angepasst werden. Dank unserer Vielseitigkeit im medizinischen Umfeld und unserem Kundenstamm von über 590 Praxen sind wir ein optimaler Partner für Sie.

\section{Kern Concept AG, Gossau SG (AESKULAP)} AESKULAP ist ein extrem schnelles WindowsProgramm, das zusammen mit einer SQL-Datenbank höchste Verarbeitungsgeschwindigkeit, Kompaktheit und schnellste Zugriffsmöglichkeiten gewährleistet. AESKULAP bietet für alle Bedürfnisse eine optimale und intelligente Lösung: vom einfachen Abrechnungssystem mit sämtlichen Leistungserfassungsmöglichkeiten bis zur vollstrukturierten elektronischen KG-Führung, die in der Praxis keine Wünsche offenlässt. Rund 200 Praxen arbeiten bereits mit der elektronischen KG-Führung. AESKULAP ist ein modular aufgebautes, anpassbares System mit einer Vielzahl von Modulen.

\section{Triamun AG, Gümligen (TriaMed)}

Triamun entwickelt und vermarktet innovative Softwarelösungen für Praxis- und Apothekenmanagement. Individuelle Beratung, die gesamte Soft- und Hardware, ein umfassender Support und Schulung aus einer Hand. Die Produkte und Dienstleistungen auf den beiden Plattformen ASP und .NET bieten für jedes Bedürfnis die geeignete Lösung. Die Softwarelösungen auf der ASP-Plattform für Apotheken und Arztpraxen basieren auf browsergestützter Technologie und wurden zusammen mit Ärzten und Apothekern entwickelt. So sind intuitiv bedienbare Managementlösungen für Apotheken und Praxen entstanden, die dem Bedürfnis nach zentralem Outsourcing Rechnung tragen. Die Softwarelösungen auf der .NET-Plattform, mit ihren Zusatzmodulen für die Arztpraxis, wurden während 20 Jahren den Kundenbedürfnissen ständig angepasst, so dass sie heute als modernste lokale Client-Server-Lösung oder zentrale TerminalServer-Lösung optimal auf die Abläufe in der Arztpraxis zugeschnitten sind.

\section{TMR - Triangle Micro Research AG,} Hölstein (WinMed ${ }^{\circledR}$ )

Die TMR AG wurde von Ärzten, Wissenschaftlern, Unternehmern und Dozenten der Uni Basel 1993 als «Spin-off»-Firma gegründet. Den Schwerpunkt in der Entwicklung der TMR AG bilden Anwendungen im Bereich der medizinischen Telekommunikation, Internet sowie der mehrmandanten- und mehrplatzfähigen Ärztesoftware WinMed $^{\circledR}$. WinMed ${ }^{\circledR}$ ist ein vollständiges, äusserst einfach zu bedienendes Arztpraxisadministrationssystem mit integrierter Kommunikationsplattform, Bild- und Dokumentenverwaltung sowie modernster elektronischer Krankengeschichte. WinMed ${ }^{\circledR}$ wird nach ausgereifter mehrjähriger Pilotphase erfolgreich seit 1998 verkauft und zählt heute zu den meistgekauften Ärztesoftwarepaketen. Vertrieben wird WinMed ${ }^{\circledR}$ in der Deutschschweiz von TMR AG selbst und im Tessin von GFP Mediconsul in Massagno.

\section{vitodata AG, Ohringen b. Winterthur (vitoMed-Administrationssystem)}

Die vitodata AG besteht seit mehr als 26 Jahren. Die Unternehmung konzentriert sich auf Praxisund Kliniklösungen. Die innovative Haltung eröffnet laufend neue Einsatzgebiete - immer mit dem entsprechenden Nutzen für die Anwender. In den ersten 25 Jahren stand die Abrechnung mit der ICT im Vordergrund. Heute ist die vitodata AG auch bei der elektronischen Krankengeschichtenführung an der Spitze im Schweizer Markt. Die Marktführerschaft ist für das ganze Team der vitodata AG eine Verpflichtung, im Sinne des Investitionsschutzes für die Kunden den Fortbestand zu sichern und unternehmerisch und ethisch korrekt zu handeln. 


\section{Röntgen in der Arztpraxis}

Themen (Details $\rightarrow$ www.fmhservices.ch) Vom konventionellen zum digitalen Röntgen Rentabilität Röntgen in der Arztpraxis - Neue Vorschriften ab 1. Januar 2008 - Evaluation und Beschaffung neuer oder gebrauchter Anlagen Komplette Marktübersicht mit Preisen und Leistungskomponenten.

\section{Kosten}

Fr. 300.- (inkl. sämtlicher Kursunterlagen und Verpflegung).

\section{Daten}

K19 Donnerstag, 22. November 2007 Wiroma AG Niederscherli BE $9.30-16.00 \mathrm{Uhr}$

\section{Ouverture et reprise d'un cabinet médical}

Contenu (Détails $\rightarrow$ www.fmhservices.ch)

Business plan - Aménagement - Estimation d'inventaire et goodwill d'un cabinet - Administration - Assurances - Aspects juridiques et fiscaux - Passage du statut de salarié à celui d'indépendant et fiscalité.

\section{Sponsors}

Assurance des Médecins Suisses, Caisse-Maladie des Médecins Suisses, Unilabs SA, Viollier SA et EMH Editions médicales suisses $\mathrm{SA}$.

\section{Dates}

$\begin{array}{lll}\text { K23 Jeudi } 22 \text { novembre } 2007 & \text { Hôtel Europe } \\ \text { Sion } & 17.00-21.00 \mathrm{~h} & \end{array}$

\section{Remise d'un cabinet médical}

\section{Contenu (Détails $\rightarrow$ www.fmhservices.ch)}

Aspects juridiques - Estimation d'inventaire et goodwill d'un cabinet - Assurances - Conséquences fiscales d'une remise.

\section{Sponsor}

Assurance des Médecins Suisses, Caisse-Maladie des Médecins Suisses et EMH Editions médicales suisses SA.

\section{Dates}

K25

Jeudi 15 novembre 2007

Genève $17.00-20.45 \mathrm{~h}$

Hôtel Epsom

\section{Anmeldung und Auskunft / Inscription et information}

Internet www.fmhservices.ch oder FMH Consulting Services, Sandra Stadelmann, Burghöhe 1, 6208 Oberkirch, Tel. 04192500 77, Fax 041 9210586.

\section{Hinweis/Remarque}

Bei sämtlichen Seminaren, bei denen die Kosten teilweise oder gänzlich von Seminarsponsoren gedeckt werden, werden die Teilnehmeradressen den jeweiligen Sponsoren zur Verfügung gestellt. Les adresses des participants aux séminaires dont les coûts sont couverts en partie ou totalement par des sponsors sont communiquées aux sponsors concernés.

\section{Annullierungsbedingungen / Conditions d'annulation}

Bei Abmeldungen oder Fernbleiben werden folgende Unkostenbeiträge erhoben:

Un montant est perçu pour une absence ou une annulation. Il est de:

- Fr. 50.- pro Person ab 14 Tage vor Seminarbeginn / par personne dans les 15 jours avant;

- Fr. 80.- pro Person ab 7 Tage vor Seminarbeginn oder Fernbleiben / par personne dans les 7 jours avant le début du séminaire. 